IUCrJ

ISSN 2052-2525

BIOLOGY|MEDICINE

Received 25 January 2019

Accepted 3 April 2019

Edited by K. Moffat, University of Chicago, USA

₹ These authors contributed equally to this study

Keywords: S-SAD; native SAD; microcrystals; microdiffraction; radiation damage; multiple crystals; anomalous diffraction; low-energy X-rays.

PDB reference: thaumatin, 6o8a

Supporting information: this article has supporting information at www.iucrj.org

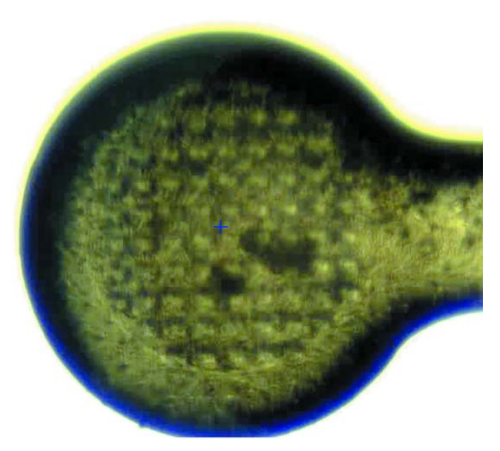

OPEN $\odot$ ACCESS

\section{Synchrotron microcrystal native-SAD phasing at a low energy}

\author{
Gongrui Guo, ${ }^{\mathrm{a}, \mathrm{b}} \neq$ Ping Zhu, ${ }^{\mathrm{a}} \neq$ Martin R. Fuchs, ${ }^{\mathrm{b}}$ Wuxian Shi, ${ }^{\mathrm{b}}$ Babak Andi, ${ }^{\mathrm{b}}$ Yuan \\ Gao, ${ }^{\mathrm{b}}$ Wayne A. Hendrickson, ${ }^{\mathrm{c}, \mathrm{d}}$ Sean McSweeney ${ }^{\mathrm{b}}$ and Qun Liu ${ }^{\mathrm{a}, \mathrm{b} *}$
} ${ }^{\mathbf{a} B i o l o g y}$ Department, Brookhaven National Laboratory, Upton, NY 11973, USA, ${ }^{\mathbf{b}}$ Photon Science, NSLS-II, Brookhaven
National Laboratory, Upton, NY 11973, USA, ${ }^{\mathbf{D}}$ Department of Biochemistry and Molecular Biophysics, Columbia
University, New York, NY 10032, USA, and ${ }^{\mathbf{d}}$ Department of Physiology and Cellular Biophysics, Columbia University,
New York, NY 10032, USA. *Correspondence e-mail: qunliu@bnl.gov

De novo structural evaluation of native biomolecules from single-wavelength anomalous diffraction (SAD) is a challenge because of the weakness of the anomalous scattering. The anomalous scattering from relevant native elements primarily sulfur in proteins and phosphorus in nucleic acids - increases as the $\mathrm{X}$-ray energy decreases toward their $K$-edge transitions. Thus, measurements at a lowered X-ray energy are promising for making native SAD routine and robust. For microcrystals with sizes less than $10 \mu \mathrm{m}$, native-SAD phasing at synchrotron microdiffraction beamlines is even more challenging because of difficulties in sample manipulation, diffraction data collection and data analysis. Native-SAD analysis from microcrystals by using X-ray free-electron lasers has been demonstrated but has required use of thousands of thousands of microcrystals to achieve the necessary accuracy. Here it is shown that by exploitation of anomalous microdiffraction signals obtained at $5 \mathrm{keV}$, by the use of polyimide wellmounts, and by an iterative crystal and frame-rejection method, microcrystal native-SAD phasing is possible from as few as about 1200 crystals. Our results show the utility of low-energy native-SAD phasing with microcrystals at synchrotron microdiffraction beamlines.

\section{Introduction}

Anomalous diffraction can produce the phase information needed to determine crystal structures, and the methods of multi- and single-wavelength anomalous diffraction (MAD and $\mathrm{SAD}$ ) now predominate for de novo structure determination of biological macromolecules (Hendrickson, 2014). Historically, conventional heavy-atom derivatizations and selenomethionine substitutions in proteins have generated the phasing elements in such analyses; however, even the light elements present in virtually all biomolecules, sulfur $(Z=16)$ in proteins and phosphorus $(Z=15)$ in nucleic acids, were shown quite early to suffice in favorable cases (Hendrickson \& Teeter, 1981; Dauter et al., 1999; Liu et al., 2000; Yang et al., 2003). Obtaining structures without the need for heavy atoms is appealing but the weakness of anomalous signals from light elements has complicated the generality of the approach. With technical advances such as pixel array detectors (Broennimann et al., 2006), rational treatment of multiple crystals (Liu et al., 2012, 2013; Akey et al., 2014), multi-axis data collection (Weinert et al., 2015) and improved structure-determination methods (de La Fortelle \& Bricogne, 1997; Sheldrick, 2010; Bunkóczi et al., 2015; Terwilliger et al., 2016), SAD phasing from native biomolecules (native SAD) is becoming routine and robust (Dauter, 2006; Doutch et al., 2012; Garman, 2014; 
Liu \& Hendrickson, 2015; Rose et al., 2015; Rose \& Wang, 2016; Liu \& Hendrickson, 2017).

Successful exploitation of native SAD for micron-sized crystals remains a particular challenge. Because of radiationdamage limitations, many microcrystals are required to obtain a complete set of diffraction data, and complications from merging of multi-crystal data exacerbate the extraction of intrinsically weak anomalous signals from microcrystal diffraction patterns. X-ray free-electron lasers are well suited for microcrystals (Chapman et al., 2011; Boutet et al., 2012). These 'super-brilliant' beams make 'diffraction before destruction' possible and enable serial femtosecond crystallography (SFX) (Chapman et al., 2011; Boutet et al., 2012). Successful SFX SAD has been demonstrated from the relatively strong anomalous signals of higher $Z$ elements, including $\operatorname{Gd}(Z=64)$ (Barends et al., 2014), $Z n(Z=30)$ (Hunter et al., 2016) and $\mathrm{Hg}(Z=80)$ (Yamashita et al., 2017). SFX SAD phasing has also been demonstrated for native, low$Z$ elements like sulfur $(Z=16)$ or sulfur plus chlorine $(Z=17)$ in tests with lysozyme (Nakane et al., 2015), thaumatin (Nass et al., 2016) and the $\mathrm{A}_{2 \mathrm{~A}}$ adenosine receptor (Batyuk et al., 2016). These native-SAD experiments were conducted at $7 \mathrm{keV}$, $6 \mathrm{keV}$ and $6 \mathrm{keV}$, respectively, and they were evaluated from 179574, 363000, and 578620 indexed images (crystals), respectively. Because of the requirement of a large sample quantity and limitations on access, SFX native-SAD phasing is time-consuming, expensive and not accessible to most users.

Synchrotron beamlines optimized for microdiffraction have become available for routine user access (Perrakis et al., 1999; Flot et al., 2010; Smith et al., 2012; Fuchs et al., 2016; Yamamoto et al., 2017). Microcrystal synchrotron crystallography is now becoming increasingly attractive at microdiffraction beamlines (Ji et al., 2010; Zeldin et al., 2013a; Gati et al., 2014; Stellato et al., 2014; Botha et al., 2015; Coquelle et al., 2015; Nogly et al., 2015; Beyerlein et al., 2017; Diederichs \& Wang, 2017; Martin-Garcia et al., 2017; Meents et al., 2017; Gao et al., 2018). Using crystals of $10-20 \mu \mathrm{m}$ in size, synchrotron SAD phasing has been demonstrated with higher $Z$ elements including iodine $(Z=53)$ and selenium $(Z=34)$ as well as for low- $Z$ native-SAD (Melnikov et al., 2017; Huang et al., 2018). With microcrystals of less than $10 \mu \mathrm{m}$, the weakness of their diffraction and challenges in sample manipulation and diffraction analysis so far have prevented practical applications at synchrotron sources.

We and others have shown the impact of crystal sizes on native SAD and suggested using low energy for optimizing microcrystal native-SAD analysis (Liu et al., 2014; Liebschner et al., 2016; Wagner et al., 2016; Guo et al., 2018). With crystals smaller than $10 \mu \mathrm{m}$, sample absorption of low-energy X-rays (3-5 keV) can be tolerated so as to permit enhancement of the imaginary component of anomalous diffraction signals (denoted as $\mathrm{f}^{\prime \prime}$ ) from sulfur. Here, we describe a microcrystal native-SAD experiment at the Frontier Microfocusing Macromolecular Crystallographic Beamline (17ID-2, FMX) at the National Synchrotron Light Source II (NSLS-II). By collecting multi-crystal data at an energy of $5 \mathrm{keV}(\lambda=2.48 \AA)$ from crystals on low-absorbance polyimide wellmounts, and by using an iterative outlier rejection strategy, we demonstrate that structure determination by synchrotron-based microcrystal native-SAD is feasible from 32323 diffraction patterns, which were collected from fewer than 1200 thaumatin microcrystals.

\section{Methods}

\subsection{Sample preparation}

Microcrystals of thaumatin were prepared and handled as previously reported (Guo et al., 2018). Briefly, microcrystals were filtrated three times through an $8 \mu \mathrm{m}$ Whatman Nuclepore Track-Etched membrane (GE Healthcare). Microcrystals were concentrated by centrifugation, and supernatant was removed. Crystal slurries were loaded to custom-made polyimide micro-sized wellmounts with $2 \mu \mathrm{m}$ holes for solvent to pass through. Solvents were then removed by touching the bottom side of the mounts using a fine-tip filter paper followed by manually plunging into liquid nitrogen for rapid freezing. Cryoprotectant was not added prior to freezing.

\subsection{Microdiffraction data collection}

Microdiffraction data were collected at the FMX beamline at NSLS-II (Fuchs et al., 2016). The beamline is equipped with an EIGER 16M detector which was calibrated to cover lowenergy X-rays. We tuned the X-ray energy to $5 \mathrm{keV}$ with a beam size of $5 \times 9 \mu \mathrm{m}$. The estimated beam flux was about $6 \times$ $10^{10}$ photons s $^{-1}$. We collected all data sets in a way similar to that implemented in the MeshAndCollect method (Zander et al., 2015). We aligned the wellmounts using a side view; and then rotated the mounts by $90^{\circ}$ so that their surface was perpendicular to the X-rays [Fig. 1(a)]. We used raster scanning with a raster step size of $5 \mu \mathrm{m}$ to find positions where diffraction data were collected. These positions, their diffraction intensities encoded as a green heat map, were visually checked for their diffraction quality and were used to guide the manual selection of positions to be queued for data collection [Fig. 1(b)]. From each position, we collected a partial rotation data set of 100 frames using rotation steps of $0.2^{\circ}$ and an exposure time of $0.02 \mathrm{~s}$ per frame. The estimated cumulative dose is $5 \mathrm{MGy}$ per crystal as calculated by the program RADDOSE-3D (Zeldin et al., 2013b). At a sampleto-detector distance of $140 \mathrm{~mm}$, the corresponding Bragg spacing is about $3.0 \AA$ at the detector edge. We collected a total of 1381 partial data sets from 18 wellmounts loaded with microcrystal slurries. On average, we collected 77 data sets on a single wellmount.

\subsection{Data reduction and assembly}

Single-crystal data sets were indexed and integrated independently by using DIALS (Waterman et al., 2016) and then scaled and merged to $2.6 \AA$ spacings by using CCP4 programs POINTLESS and AIMLESS (Evans et al., 2011; Evans \& Murshudov, 2013). Our established method was used for outlier rejection and data assembly (Guo et al., 2018). We processed single-crystal data sets as accumulated wedges of 10 , $20, \ldots, 100$ frames, ending up with 10 wedges per crystal. 


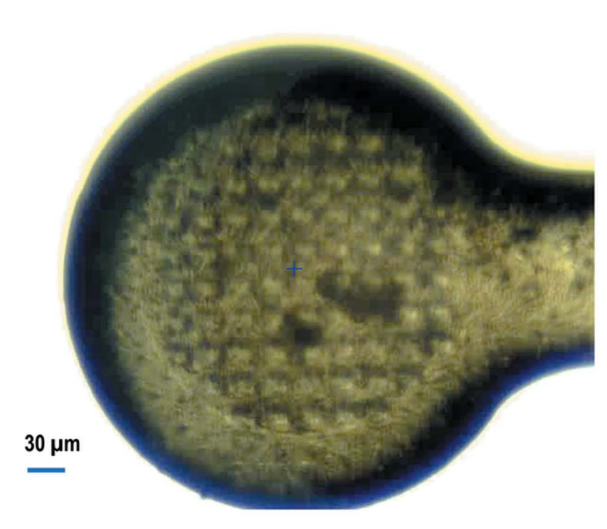

(a)

Figure 1

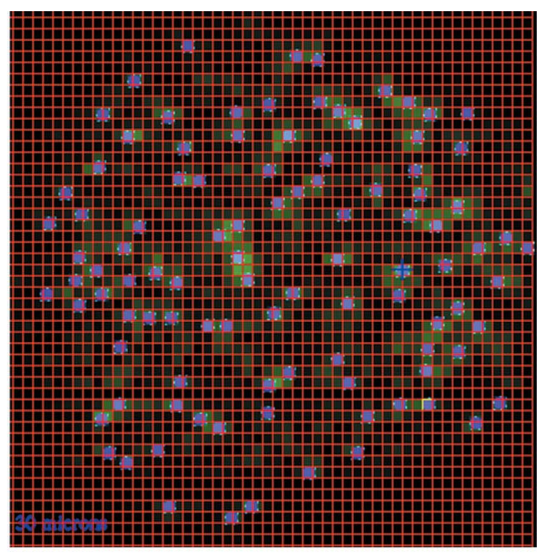

(b)

Microcrystal handling and diffraction data collection. (a) A representative image of a micro-sized wellmount loaded with microcrystals prior to a raster scanning. (b) Positions selected for data collection based on a raster-scanning heat map (green). Red meshes show the grid $(55 \mu \mathrm{m})$ used for the scan. For this example, 101 positions (purple dots) were queued for data collection.

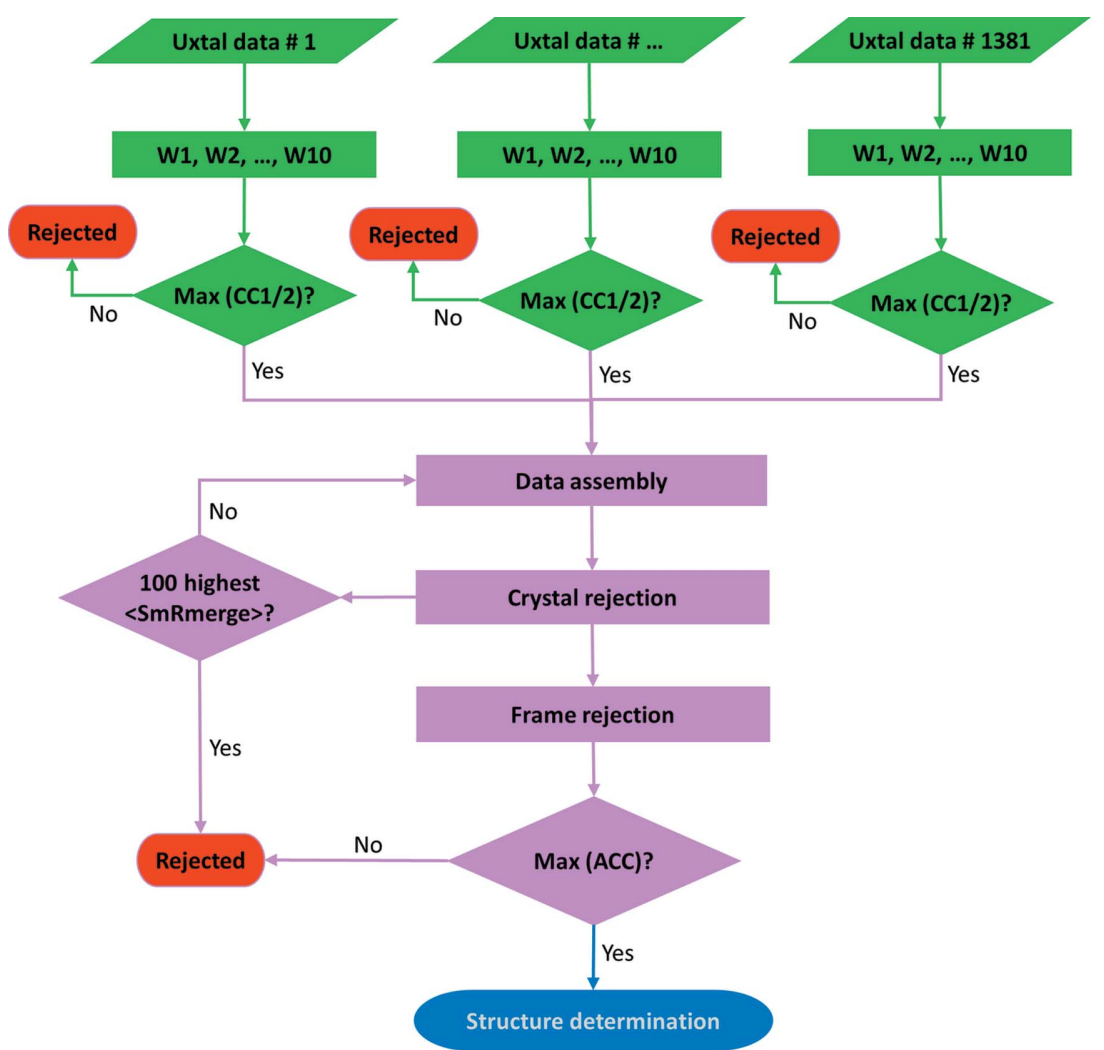

Figure 2

Schematic workflow of data assembly. Firstly, individual microcrystal data sets (100 frames each) were split and processed into ten accumulated wedges; and wedges with maximum $\mathrm{CC}_{1 / 2}$ were selected for data assembly. Secondly, iterative crystal rejection was performed by rejection of 100 microcrystals with the highest average SmRmerge, $\langle$ SmRmerge $\rangle$. After each crystal rejection, frame rejection was performed to remove outliers based on defined SmRmerge cutoffs. Finally, the assembled data set with maximum ACC was used for structure determination.

Wedges with maximum $\mathrm{CC}_{1 / 2}$ calculated at $4 \AA$ were chosen for subsequent data assembly. Smoothed frame $R_{\text {merge }}$ (SmRmerge) as reported in AIMLESS (Evans \& Murshudov, 2013) was used to score compatibility at both crystal and frame levels to guide further outlier rejections during data assembly. At the crystal level, average SmRmerge, 〈SmRmerge $\rangle$, was used to score each crystal and iterative crystal rejection was performed by rejecting 100 crystals with the highest 〈SmRmerge〉. The assembly of all 1381 single-crystal partial data sets was called 'assembled data set 1381'. 〈SmRmerge $\rangle$ was calculated at each cycle to identify the most incompatible crystals for rejection in successive cycles. Starting from assembled data set 1381, we rejected 100 crystals with the highest SmRmerge $\rangle$ and obtained assembled data set 1281 , from which updated 〈SmRmerged〉 values were calculated for preparation of another cycle of rejection. By iteration, assembled data sets 1181 , 1081, etc. were obtained until reaching the end with assembled data set 81 . So at the crystal level, a total of 14 assembled data sets were obtained. At the frame level, we used SmRmerge per frame to reject frames based on radiation-induced decay. We rejected frames at different cutoffs as defined by frame_cutoff $=[\mathrm{min}($ SmRmerge $)$ $\times(1+$ decay $)]$, where $\min ($ SmRmerge $)$ is the lowest SmRmerge within a single-crystal data set; and decay is a rejection ratio of none (effectively, decay $=\infty), 500 \%, 200 \%, 150 \%$, $100 \%$ or $50 \%$. At each rejection ratio, frames with SmRmerge larger than frame_cutoff were excluded from assembly in AIMLESS. For example, a rejection ratio at $150 \%$ indicates that frames with a SmRmerge of $150 \%$ more than $\min$ (SmRmerge) are rejected from scaling and merging. We performed frame rejection at six ratios for each of the 14 assembled data sets (assembled data sets 1381, 1281, ..., 81) and obtained a total of 84 assembled data sets with different extents of frame rejection. A schematic of our data assembly workflow is shown in Fig. 2. After crystal and frame rejection, the assembled data set 1181 with a frame-rejection ratio at $150 \%$ shows the highest anomalous correlation coefficient (ACC) (see details in results). The data-collection and refinement statistics for this assembled data set are shown in Table 1.

\subsection{Structure determination}

Substructures were determined with SHELXD (Sheldrick, 2010). 5000 SHELXD trials were performed to search for nine 
Table 1

Data-collection and refinement statistics for the merged data set 1181 with $150 \%$ frame rejection.

Values in parentheses are for the highest resolution range.

\begin{tabular}{ll}
\hline Data collection & \\
\hline Beamline & FMX (NSLS-II) \\
Wavelength $(\AA)$ & 2.48 \\
Space group & $P 4_{1} 2_{1} 2$ \\
Cell dimensions $a, c(\AA)$ & $57.70,150.45$ \\
Solvent content $(\%)$ & 53.0 \\
Bragg spacings $(\AA)$ & $40-2.63(2.70-2.63)$ \\
Total reflections & 2721845 \\
Unique reflections $\dagger$ & $8435(14853)$ \\
Completeness $(\%)$ & $100.0(100.0)$ \\
$\langle I / \sigma(I)\rangle$ & $22.4(2.1)$ \\
$R_{\text {split }}$ & $0.05(0.583)$ \\
Multiplicity & $322.7(46.8)$ \\
CC $C_{1 / 2}$ & $0.996(0.684)$ \\
Refinement & \\
Resolution $(\AA)$ & 2.6 \\
No. reflections & 8379 \\
$R_{\text {work }} / R_{\text {free }}$ & $0.186 / 0.216$ \\
No. atoms & 1654 \\
Wilson $B$ factor $\left(\AA^{2}\right)$ & 24.1 \\
Average $B$ factor $\left(\AA^{2}\right)$ & 23.7 \\
R.m.s deviations & \\
Bond length $(\AA)$ & 0.002 \\
Bond angle $\left({ }^{\circ}\right)$ & 0.435 \\
PDB code & $608 \mathrm{a}$
\end{tabular}

$\dagger$ In parentheses is the number of unique reflections with Bijvoet pairs separated.

anomalous scatterers with $E_{\text {min }}$ cutoffs between 1.3 and 1.7 and with resolution cutoffs between 3.5 and $4.2 \AA$. The best substructure was used for calculating initial SAD phases in PHASER (Read \& McCoy, 2011) or SHARP (Vonrhein et al., 2007), followed by density modification with $D M$ or SOLOMON (Cowtan \& Zhang, 1999). Automatic model building was performed using BUCCANEER (Cowtan, 2006). Further iterative model building and refinement were performed in COOT and PHENIX.REFINE, respectively (Afonine et al., 2012; Echols et al., 2014). Bijvoet pairs were treated as two different reflections in all refinements, and the resultant Fourier coefficients were used for calculation of Bijvoet-difference Fourier maps. The stereochemistry of the refined structure was validated with PROCHECK (Laskowski et al., 1993) and MolProbity (Chen et al., 2010) for quality assurance. The refinement statistics for the assembled data set 1181 with $150 \%$ frame rejection are listed in Table 1.

\section{Results}

\subsection{Single-crystal data sets}

The required X-ray dose for a given signal-to-noise ratio is inversely proportional to the volume of microcrystals (Holton \& Frankel, 2010). Therefore, to obtain diffraction spots to the required Bragg spacings, only an incomplete partial data set could be collected from each microcrystal before its being killed by radiation damage. We used $\mathrm{CC}_{1 / 2}$ at $4.0 \AA$ to evaluate single-crystal data quality and did initial rejection to remove frames which caused the decrease of $\mathrm{CC}_{1 / 2}$ if they were combined. By this initial frame rejection at a lower resolution, we made a compromise of having more frames to be selected for downstream assembly, while rejecting frames with too much radiation damage which would make the scaling process unstable if they were not rejected. Fig. 3(a) shows the distribution of microcrystals with respect to $\mathrm{CC}_{1 / 2}$ after the initial frame rejection. With the maximum $\mathrm{CC}_{1 / 2}$ as criteria, we can select 20 to 100 frames per crystal, indicating that single crystals respond differently to radiation damage, with 30 to 40 frames selected for most single crystals [Fig. 3(b)]. After the initial frame rejection, we selected a total of 51570 frames from 1381 crystals for subsequent data assembly. The diffraction power of these microcrystals is weak. Necessarily the average $I / \sigma($ average $I),\langle I / \sigma(I)\rangle$, of these single-crystal data sets tends to be low. For the 1381 single-crystal data sets, most display an $\langle I / \sigma(I)\rangle$ of about 2 [Fig. 3(c)].

Crystal morphology and distribution on the support could impact the reciprocal-space coverage and data assembly. We used patterned microwells to distribute the orientations of microcrystals on the support. To check the orientation distribution of the microcrystals, we indexed single-crystal data sets, converted their orientation matrices to three Euler angles and plotted the angular distribution in Fig. 3(d). Euler angles $\alpha$ and $\gamma$ have a roughly uniform distribution between 0 and $180^{\circ}$ but there is a skewed distribution for $\beta$ peaked at $\sim 80^{\circ}$. Considering that the lattice of thaumatin crystals is tetragonal, the skewed distribution of $\beta$ can be well accommodated by symmetry-related measurements.

\subsection{Assembled data sets}

We combined the 1381 partial single-crystal data sets and rejected outlier crystals and frames progressively. Because of data incompleteness in each single-crystal data set and missing cross-crystal reflections for a reliable correlation analysis, we used SmRmerge which indicates the overall compatibility of each frame to the merged data set. By using 〈SmRmerge $\rangle$, we sorted the 1381 single-crystal data sets and iteratively rejected 100 crystals of the highest $\langle\mathrm{SmRmerge}\rangle$. In two iterations, such crystal rejection removed the 200 most statistically incompatible crystals which are detrimental to $\mathrm{CC}_{1 / 2}, R_{\text {split }}$ and ACC (assembled data sets 1281 and 1381) (Fig. 4). For each of these 14 assembled data sets, we did frame rejections at six ratios based on SmRmerge. By combination of the two rejection strategies, we found that significant anomalous signals can be extracted from 1081 or 1181 crystals with frame-rejection ratios of $150 \%$ or $200 \%$ [Fig. 4(c)]. Assembled data set 1181 with a frame-rejection ratio at $150 \%$ gave the highest ACC of $51.7 \%$. This data set was then used in structure determination. We also found that stringent frame rejections at $50 \%$ and $100 \%$ reduced anomalous signals. Because of variation among microcrystals, we suggest conducting frame rejection at different ratios and selecting the one with the highest ACC for structure analysis. 


\subsection{SAD phasing and anomalous signals}

Thaumatin contains eight disulfide bonds and one methionine residue for a total of 17 sulfur atoms. With a resolution cutoff at $4.0 \AA$, disulfide bonds $(2.02 \AA$ ) are not resolvable and we thus searched for nine sites in SHELXD. With an $E_{\text {min }}$ cutoff at 1.4 and 5000 SHELXD trials, we obtained 41 substructure solutions with the highest $\mathrm{CC}_{\mathrm{all}}$ and $\mathrm{CC}_{\text {weak }}$ of $45.7 \%$ and $22.8 \%$, respectively [Fig. 5(a)]. Substructures were used for SAD phasing using SHARP (Vonrhein et al., 2007). After density modification, electron-density maps at $2.6 \AA$ resolution were of sufficient quality for model building in COOT [Fig. 5(b)]. The program BUCCANEER (Cowtan, 2006) was able to build 178 out of 202 residues automatically. The refined structure has an $R / R_{\text {free }}$ of $18.6 / 21.6 \%$, indicating the data quality of this assembled data. In comparison to the experimental electron density, Fig. 5(c) shows the electron density of the refined map.

To evaluate the strength of anomalous signals, we carried out $\mathrm{f}$ " refinement (Liu et al., 2013) for the eight disulfide bonds and the one methionine in PHENIX.REFINE by using

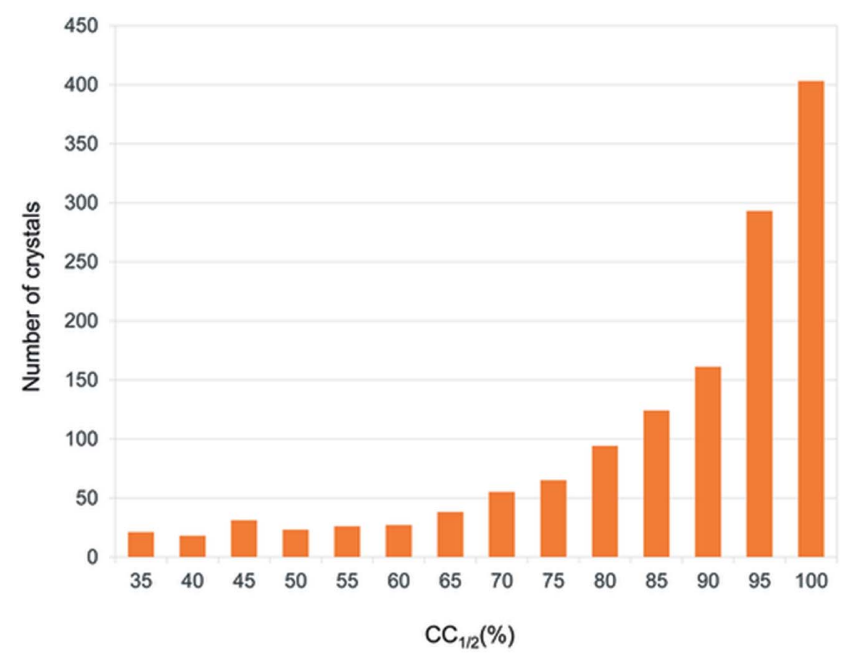

(a)

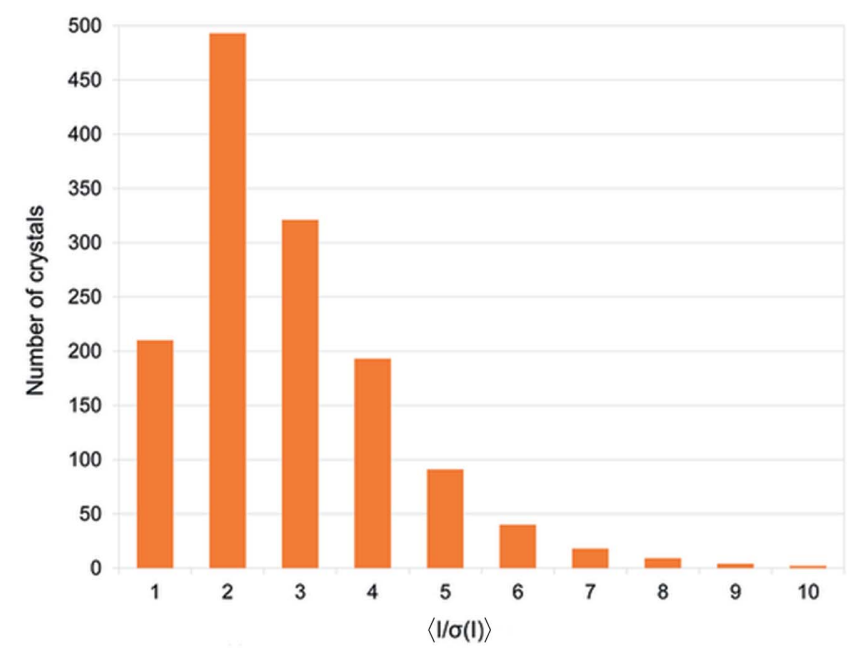

Figure 3

(c)
Bijvoet pairs. The highest $\mathrm{f}^{\prime \prime}$ value is 1.34 e for Cys149-Cys158, and the lowest $\mathrm{f}^{\prime \prime}$ is 0.96 e for Cys56-Cys66, with an average of $1.14 \mathrm{e}$ for all sulfur atoms. At $5 \mathrm{keV}$, the theoretical $\mathrm{f}^{\prime \prime}$ for sulfur is $1.31 \mathrm{e}$. Therefore, our low-energy experiment has preserved the majority of the anomalous diffraction signals. Consequently, all nine Bijvoet-difference Fourier peaks can be seen clearly beyond $3.0 \sigma$ with the highest peak at $10 \sigma$ for Cys121-Cys193 [Fig. 5(d)]. The success in structure determination and the strengths of the anomalous signals demonstrate that native SAD is possible at a synchrotron beamline from about 1200 crystals of less than $10 \mu \mathrm{m}$ in size.

\subsection{Frame rejection and radiation damage}

We explored the impact of frame-rejection ratios on assembled data set 1181 . We found that ACCs and Bijvoetdifference Fourier peaks are dramatically affected [Figs. 6(a) and $6(b)]$. With a rejection ratio of $100 \%$ or $50 \%$, i.e. with fewer damaged data being included in the assembled data set, ACC decreased substantially for low-resolution shells $\left(d_{\min }>\right.$ $5 \AA$ ). Bijvoet-difference Fourier peaks and the gap between

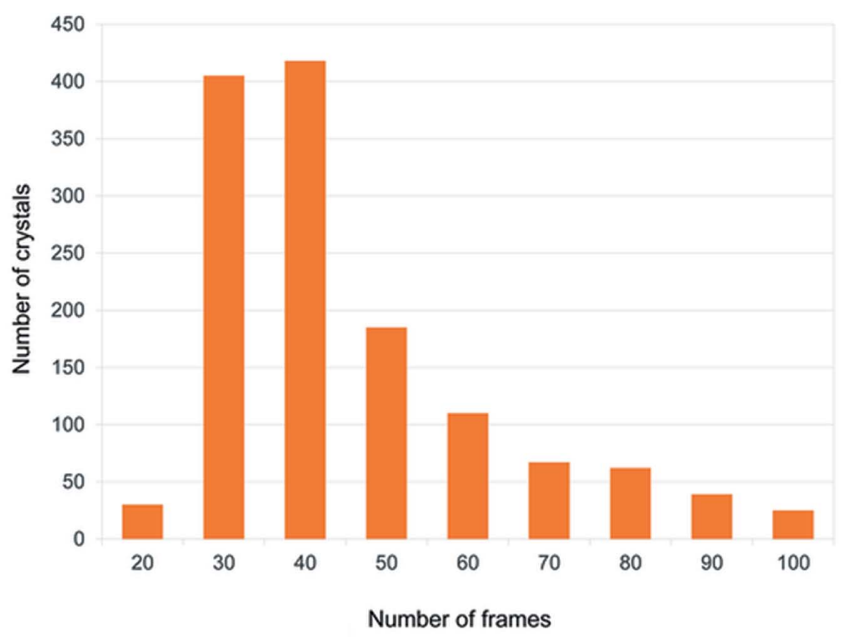

(b)

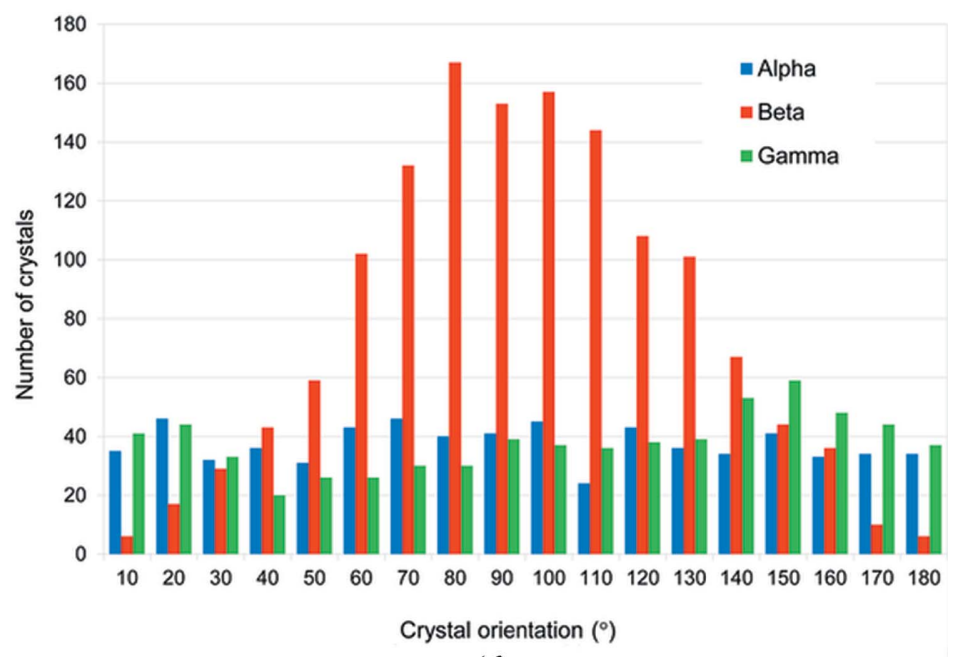

(d)

Data analysis of single-crystal data sets. (a) $\mathrm{CC}_{1 / 2}$ distribution of single crystals. (b) Distribution of the number of frames selected from single crystals. $(c)$ $\langle I / \sigma(I)\rangle$ distribution of single crystals. (d) Distribution of crystal orientations shown as three Euler angles $\alpha, \beta$, and $\gamma$. 


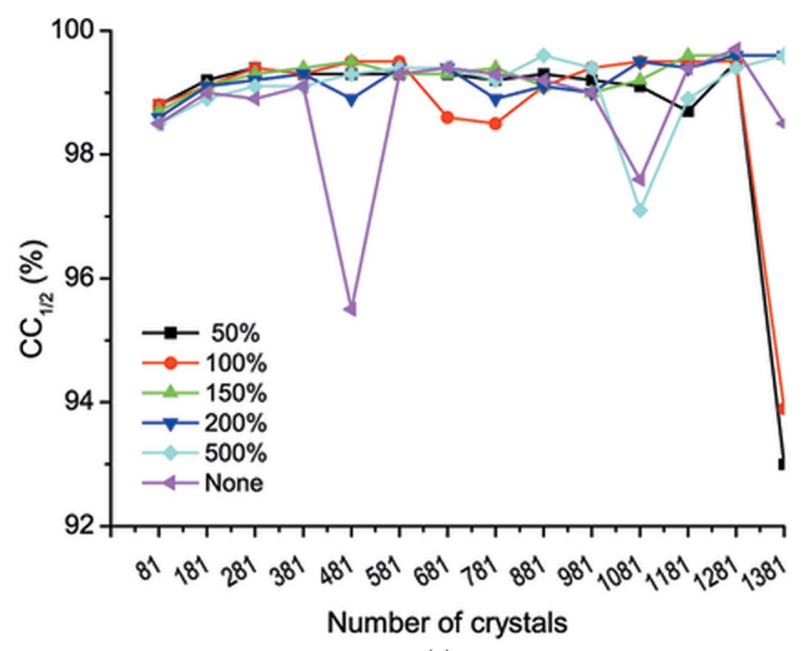

(a)

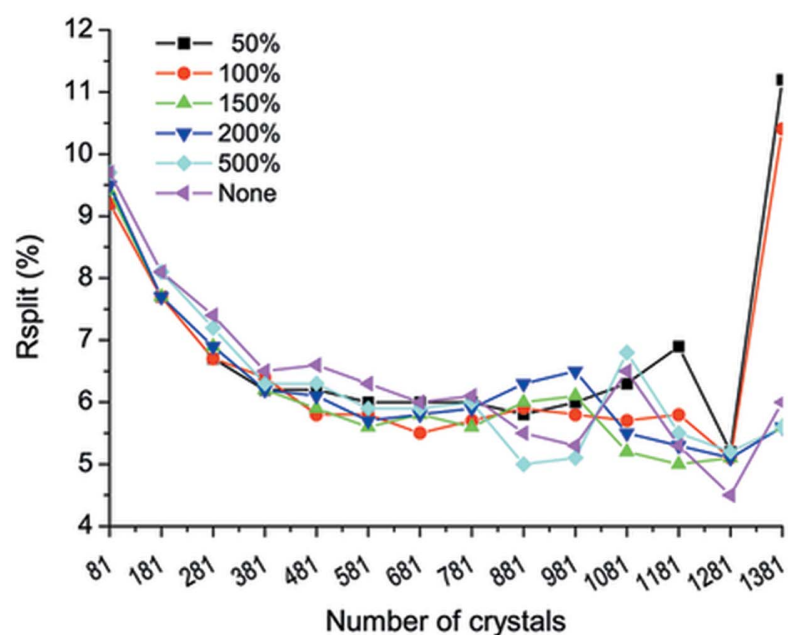

(b)

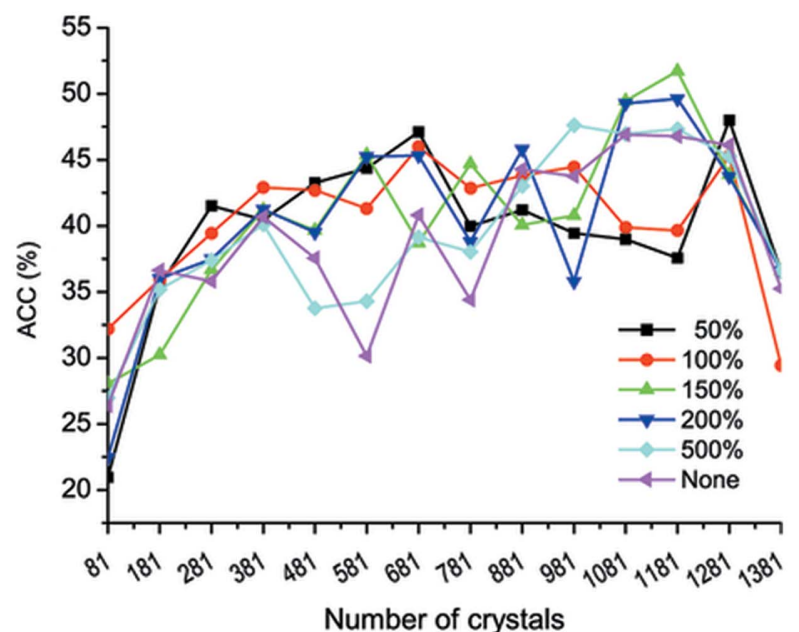

(c)

Figure 4

Data analysis of assembled data sets. (a) $\mathrm{CC}_{1 / 2},(b) R_{\text {split }}$ and (c) ACC. Within each plot, the curves are corresponding to a different extent of frame rejection after each cycle of crystal rejection. Frame rejection is shown at six different ratios with $50 \%$ being the most stringent rejection and 'None' being no frame rejection. ACC values were calculated with high-resolution data truncated to $4.0 \AA$. peak 9 (the lowest anomalous peak) and peak 10 (the highest background peak) also decreased perhaps because of deteriorated low-resolution anomalous signals. With a rejection ratio of $150 \%$ or higher, low-resolution anomalous signals were preserved with neither much variation of Bijvoetdifference Fourier peaks nor the ACC values. This observation suggests that radiation-damaged frames still contributed to the enhancement of low-resolution anomalous signals and should be preserved during data processing. We attribute such enhancement to the enhanced multiplicity, which improved the signal-to-noise ratio through averaging. In addition, radiation damage, including non-isomorphism that it may induce, affects high-angle data first. Although our dose of 5 MGy per crystal is well below the 30 MGy Garman limit (Garman \& Weik, 2017) and 20 MGy Henderson limit (Henderson, 1990), it is widely recognized that a more stringent level is required to preserve weak anomalous signals.

To further investigate the impact of frame rejection on native-SAD structure determination, we ran substructure searches by using SHELXD for assembled data set 1181 with different frame-rejection ratios. We found that preserving lowresolution anomalous signals is correlated with success in substructure determination [Fig. 6(c)]. As may be seen, with rejection ratios of $50 \%$ and $100 \%$ there are no substructure solutions. As a contrast, assembled data sets with framerejection ratios of $150 \%$ or more, including no rejection, yield substructure solutions. This result emphasizes that proper frame rejection is important for substructure determination from microcrystals.

To evaluate the effect of frame rejection on structure solvability, we used molecular-replacement SAD (MR-SAD) (Read \& McCoy, 2011) to calculate SAD phases starting with the refined structure. After density modification of SAD phases, we calculated the map correlation coefficient (mapCC) between the SAD and the model map. We found that a rejection ratio of $150 \%$ or higher gave a mapCC of $56 \%$ or more. However, assembled data sets with rejection ratios of $50 \%$ and $100 \%$ have mapCC of about $33 \%$, indicating much weaker anomalous signals arising from too stringent frame rejection, even though the radiation damage is necessarily less in these two data sets. Therefore, for microcrystal native-SAD phasing at a synchrotron with rotation data collection, proper frame rejection is necessary. It is vital to use a less stringent frame-rejection ratio in order to enhance low-resolution anomalous signals for substructure determination and phasing.

\section{Discussion}

\subsection{Microdiffraction data collection}

With microcrystals less than $10 \mu \mathrm{m}$ in size, we have demonstrated the capability of synchrotron native-SAD phasing at a lower energy of $5 \mathrm{keV}$. Our experiment used $\sim 1400$ microcrystals from which 1181 crystals were used for an optimal data assembly. To collect thousands of data sets, an efficient data-collection strategy is necessary which requires 
high-density crystal mounts with fast microcrystal identification and collection. To increase the crystal density on wellmounts, we concentrated microcrystals, removed as much supernatant as possible without touching the crystal slurry, and obtained on average 77 data sets per wellmount. The highest yield we achieved had 101 data sets on the wellmount.

We used a beam size of $5 \times 9 \mu \mathrm{m}$ for data collection. To prevent X-ray damage to nearby regions, we selected diffraction positions separated by at least one mesh grid $(5 \mu \mathrm{m}$ in the horizontal) [Fig. 1(c)]. This microdiffraction datacollection strategy could be automated as implemented at the European Synchrotron Radiation Facility (Zander et al., 2015) and the Swiss Light Source (Basu et al., 2019). Preliminary analysis of raster-scanned crystals may provide useful information to assist designing data-collection strategies, for example by preventing collecting data from overlapped crystals (Melnikov et al., 2018). Steps of crystal identification and rotation data collection could be combined as originally proposed and tested (Gati et al., 2014). This mode of data collection reduces unnecessary radiation damage but with a compromise of having a lot of empty frames that have to be identified and rejected prior to standard diffraction data analysis (Gao et al., 2018).

\subsection{Radiation damage}

In order to extract weak anomalous signals for microcrystal native-SAD phasing, radiation damage is one major challenge. To squeeze out the most data from a single crystal and subsequently to extract weak anomalous signals, we took three approaches. The first approach is to overexpose each microcrystal by collecting more frames per sample. As shown in Fig. $3(b)$, although most microcrystals only allowed for optimal data with wedges containing 30-40 frames, we collected 100 frames from which an optimal wedge could be selected. The second approach is to process single-crystal data sets progressively into successive data wedges. This assures to have

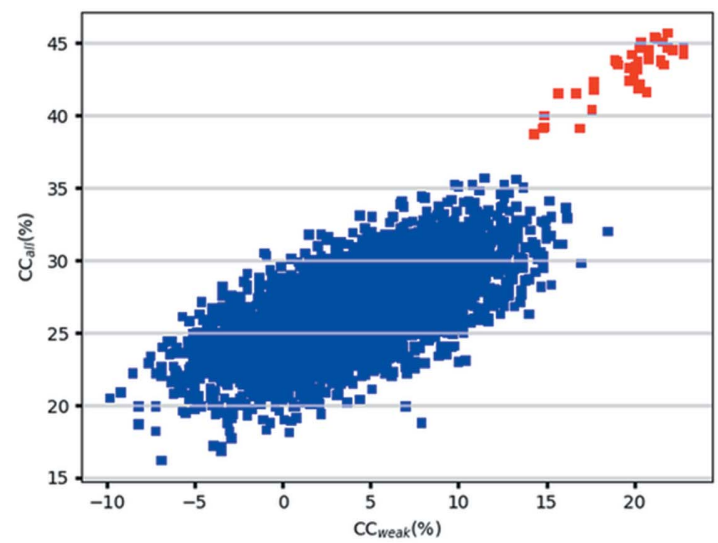

(a)

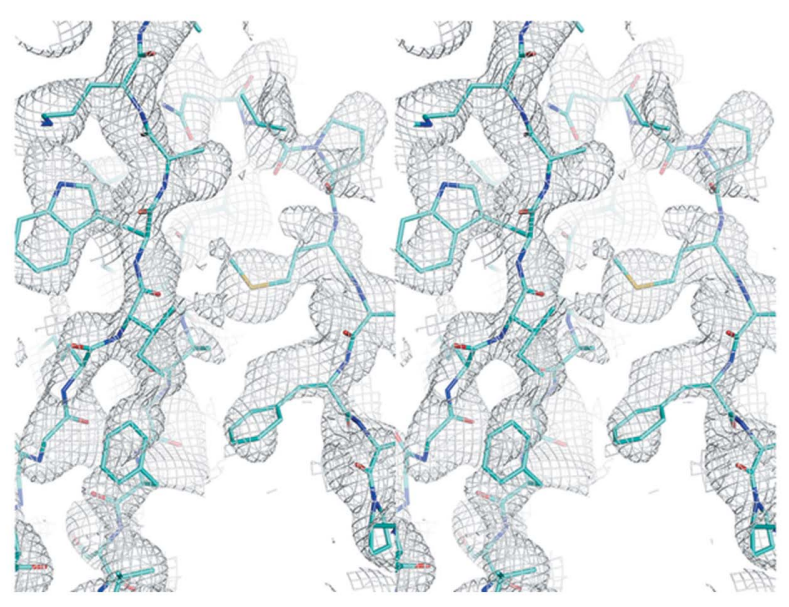

(b)

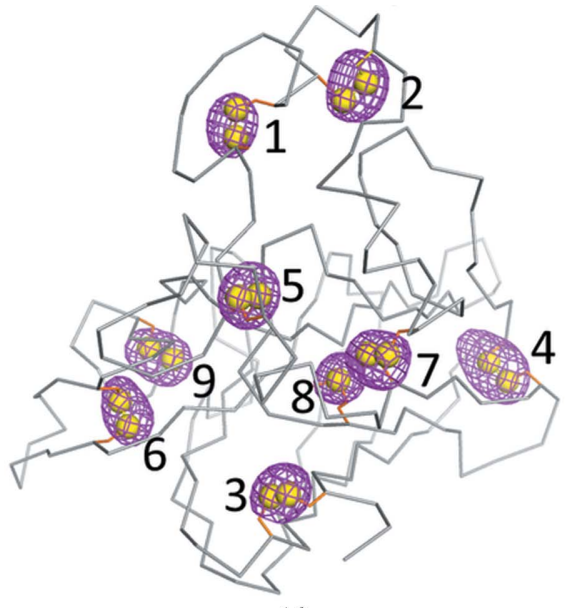

(d)

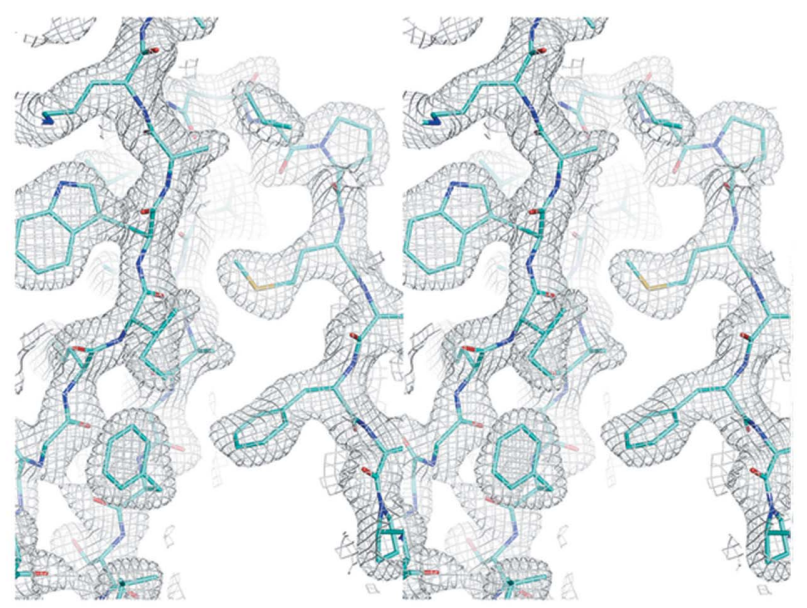

(c)

Figure 5

Structure determination and phasing. (a) $\mathrm{CC}_{\text {all }} / \mathrm{CC}_{\text {weak }}$ of 5000 SHELXD trials, (b) experimental electron density after density modification, (c) refined electron-density map and $(d)$ Bijvoet-difference Fourier peaks. Peaks for anomalous scatterers (sulfur) are shown as magenta isomeshes contoured at $3 \sigma$. The overall structure of thaumatin is shown as ribbons. The numbers and yellow spheres indicate, respectively, the positions and atoms of anomalous scatters in the structure: (1) Cys149-Cys158, (2) Cys159-Cys164, (3) Cys9-Cys204, (4) Cys121-Cys193, (5) Cys134-Cys145, (6) Cys56-Cys66, (7) Cys126Cys177, (8) Met122, (9) Cys71-Cys77. 
as many frames as possible to be included by using maximum $\mathrm{CC}_{1 / 2}$ at a lowered resolution (4 $\AA$ for thaumatin) [Fig. 3(b)]. The third strategy is to fine-tune the rejection of radiationdamaged frames after each cycle of crystal rejection (Fig. 4). We found that combining these strategies can effectively treat radiation damage while extracting the most anomalous diffraction signal from each single crystal.

Through our frame-rejection analysis, we found that including more data, even though damaged, is beneficial to enhance low-resolution anomalous signals for substructure determination. For example, on SHELXD substructure determination, the highest $\mathrm{CC}_{\mathrm{all}} / \mathrm{CC}_{\text {weak }}(45.8 \% / 27.8 \%)$ is from the assembled data set 1181 with no frame rejection. We did substructure searches using reflections out to $4.0 \AA$ spacings; while the SAD phases were calculated and density modifications were performed with all reflections out to $2.6 \AA$. In terms of mapCC, the best phases $(61 \%)$ are from $150 \%$ frame rejection.

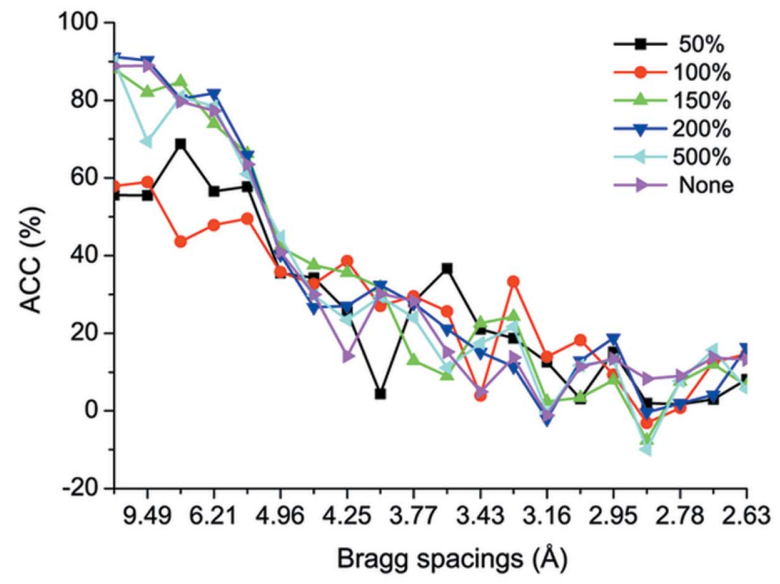

(a)

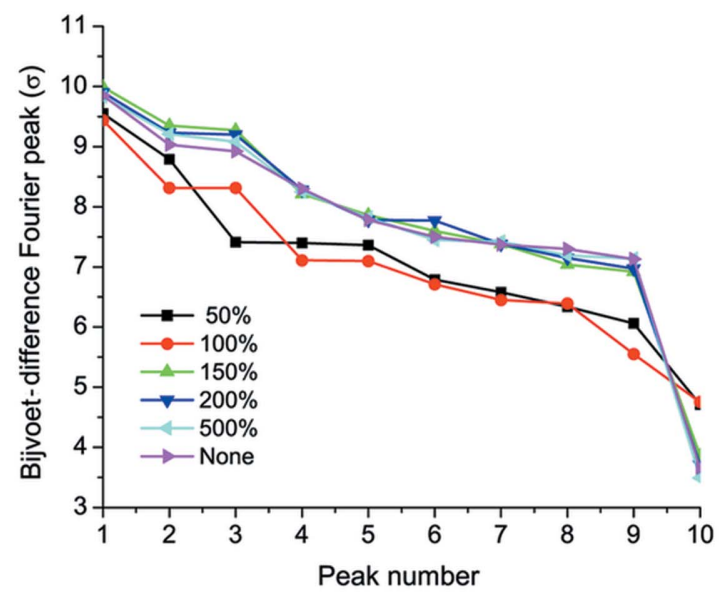

(b)
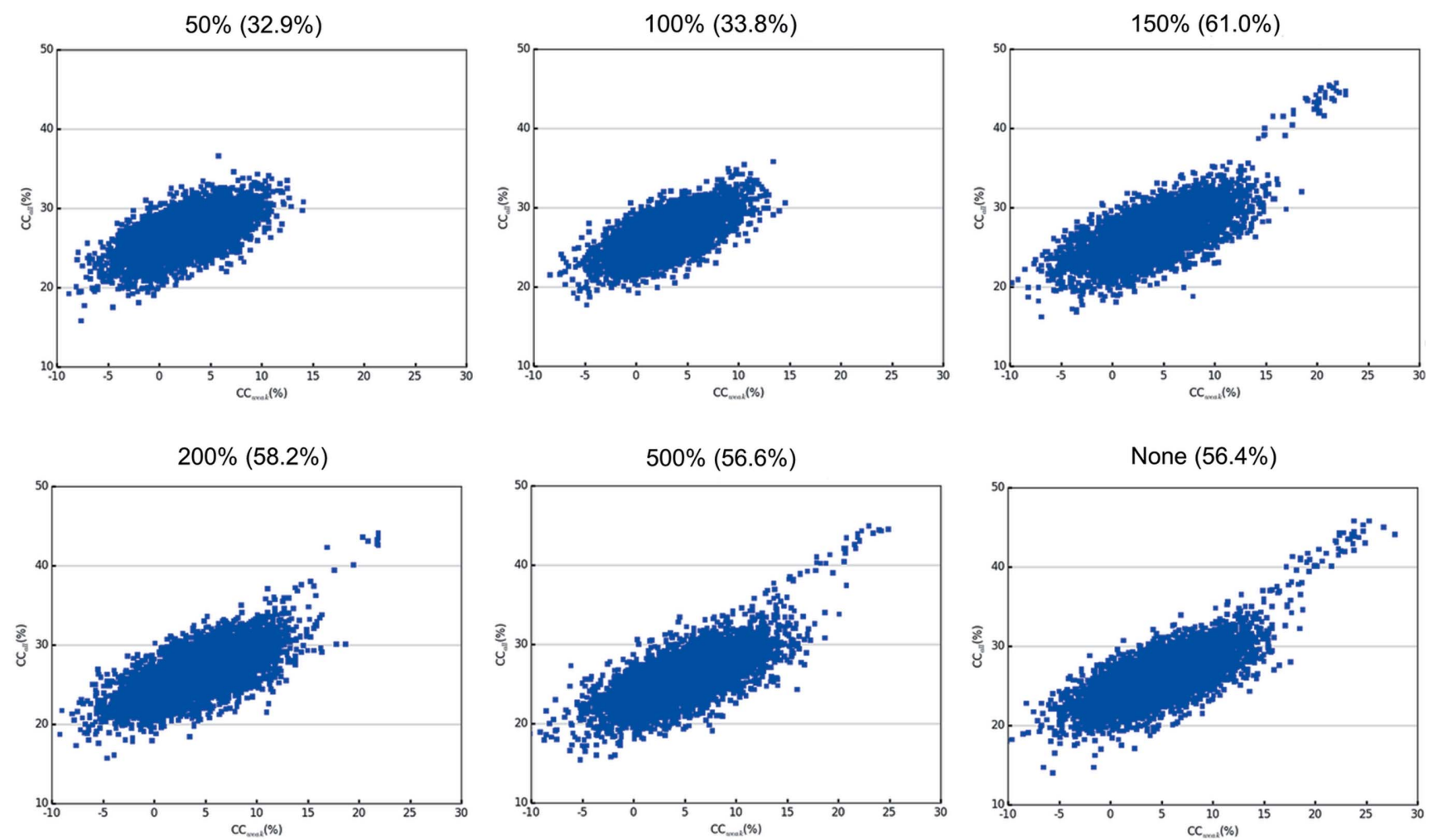

(c)

Figure 6

Analysis of the assembled data set 1181. (a) Plot of $\mathrm{ACC}(\%)$ with respect to Bragg spacings. (b) Plot of the ten highest Bijvoet-difference Fourier peaks. The first nine peaks (peaks 1-9) are from nine anomalous scatterers and the tenth peak is from noise. $(c) \mathrm{Plot}$ of $\mathrm{CC}_{\text {all }} / \mathrm{CC}$ weak from $S H E L X D$ substructure determination for data set 1181 with different frame-rejection ratios. For each data set, $5000 S H E L X D$ trials were performed with $E_{\text {min }}=1.4$ and resolution cutoff $=4.0$. MR-SAD with the known structure was used for SAD phasing and the mapCC after density modification are shown in parentheses. 


\subsection{Low-energy X-rays}

Low-energy X-rays are very attractive for native-SAD phasing because of the increased $\mathrm{f}$ " for sulfur and phosphorus in native biomacromolecules (Hendrickson, 2014; Liu \& Hendrickson, 2015). In addition, overall diffraction signals at a lower energy are much stronger, proportional to the cube of the corresponding wavelength (Holton \& Frankel, 2010). However, absorption of low-energy X-rays, including anomalous signals, also increases with decreased energy. The absorption may come from the crystal itself, the crystal support (mount), the path between crystal and detector, and the detector sensor material. In our $5 \mathrm{keV}$ experiment, we used microcrystals of a few micrometres. The sample absorption was calculated to be less than $2 \%$ and could thus be ignored. To reduce absorption from the support, we used polyimide wellmounts of about $3 \mu \mathrm{m}$ thick which can transmit $99 \%$ of X-rays at $5 \mathrm{keV}$. Therefore, our polyimide mounts are well suited for low-energy native-SAD experiments. At $5.4 \mathrm{keV}$, the specified detective quantum efficiency for the EIGER $16 \mathrm{M}$ is $94 \%$, which is close to the $5 \mathrm{keV}$ that we used. By using the setting calibrated at $5 \mathrm{keV}$, the sensor absorption should not impact our low-energy experiment. It is noted that we did not use a helium environment to reduce air absorption. Our sample-to-detector distance is $140 \mathrm{~mm}$, which may contribute to air absorption of $5 \mathrm{keV}$ X-rays by $46.3 \%$. Nevertheless, structure determination by microcrystal nativeSAD phasing proved possible at $5 \mathrm{keV}$ even without a helium environment. Instrumentation of a helium or vacuum environment would certainly facilitate native-SAD phasing at low energy as implemented at the Diamond Light Source (Wagner et al., 2016) and the Photon Factory (Liebschner et al., 2016).

Measurement at an X-ray wavelength of $2.1 \AA(E=$ $5.9 \mathrm{keV}$ ) has been proposed as optimal for native-SAD phasing, although the possible variation of this optimum with crystal size was not explored (Mueller-Dieckmann et al., 2005). By using a multi-crystal strategy, we also showed that the anomalous signals recorded from data collected at $6 \mathrm{keV}$ are superior to those collected at $7 \mathrm{keV}$ (Liu et al., 2014). In consideration of the crystal-size-dependent absorption of lowenergy X-rays, using an energy below $6 \mathrm{keV}$ has been proposed for microcrystals (Liu et al., 2014; Liebschner et al., 2016; Wagner et al., 2016). Even with relatively large crystals $(50 \mu \mathrm{m})$, it has been shown that a longer wavelength of $2.7 \AA$ $(E=4.6 \mathrm{keV})$ is superior to a shorter wavelength of $1.9 \AA(E=$ $6.5 \mathrm{keV}$ ) for native-SAD phasing (Liebschner et al., 2016). In our microcrystal native-SAD experiments at a low energy, we used an energy at $5 \mathrm{keV}$, the low-energy limit of the photon delivery system of the FMX beamline. Similar to what have been done for larger crystals, it may be interesting to explore the native-SAD phasing efficacy of microcrystals with respect to X-ray energies between 7 and $4 \mathrm{keV}$, although such a study is likely to need additional instrumentation not currently available at FMX.

\subsection{Multiple crystals and outlier rejection}

The use of multiple crystals provides an efficient way of obtaining the necessary multiplicity and data accuracy for enhancing anomalous signals for de novo structure determination (Liu et al., 2011; Liu \& Hendrickson, 2017). One major problem arising from using multiple crystals is the variation among crystals which compromises the data merging if not treated properly. We and others have proposed to use unit-cell variation, diffraction-intensity dissimilarity and relative contribution of ACCs for rejection of outlier crystals (Giordano et al., 2012; Liu et al., 2012; Foadi et al., 2013). For microcrystals, only a partial data set is achievable per crystal; thus multiple crystals must be used and outlier rejection needs to be performed based on unit-cell variation or correlation to a merged or reference data set (Axford et al., 2015; Guo et al., 2018; Yamashita et al., 2018; Basu et al., 2019). The use of genetic algorithm classification and outlier crystal rejection provide an alternative way for finding compatible partial data sets (Zander et al., 2016). In our experience, the unit-cell dimensions of thaumatin microcrystal do not vary much (Guo et al., 2018). Consequently, in our $5 \mathrm{keV}$ experiment, we did not use unit-cell variation for initial rejection of nonisomorphic data. Instead, we combined crystal and framerejection steps with the data-assembly process, thus simplifying the data-analysis workflow. By using maximum $\mathrm{CC}_{1 / 2}$ at $4 \AA$ for initial frame rejection, we also did not use the reference data set, further simplifying and speeding up data assembly. Selection of resolution cutoff at $4 \AA$ so far is empirical. Using a different maximum $\mathrm{CC}_{1 / 2}$ cutoff would affect the total number of frames being included for assembling and subsequent crystal and frame rejection. Based on our experience, one could also use several resolution cutoffs to optimize the frame selection in single-crystal data sets.

\section{Concluding remarks}

Native-SAD phasing from microcrystals of less than $10 \mu \mathrm{m}$ in size is challenging and was not yet routinely demonstrated at a synchrotron source. Here we show that native-SAD phasing from such microcrystals is feasible at a synchrotron beamline by using as few as 1200 crystals with a multiplicity of 323 . The use of low-energy X-rays at $5 \mathrm{keV}$, low-absorbance polyimide wellmounts and iterative outlier rejections make microcrystal native-SAD phasing promising for real-life applications on challenging samples such as microcrystals of membrane proteins and complexes. Native-SAD phasing with larger crystals may also benefit from such low-energy experiment and analysis.

\section{Funding information}

This work was supported in part by Brookhaven National Laboratory LDRD 17-023 and the National Institutes of Health grant GM107462. The FMX beamline is part of the Life Science Biomedical Technology Research resource which is primarily supported by the National Institutes of Health, National Institute of General Medical Sciences through a Biomedical Technology Research Resource P41 grant (P41GM111244), and by the DOE Office of Biological and 
Environmental Research (KP1605010). NSLS-II is supported in part by the U.S. Department of Energy, Office of Science, Office of Basic Energy Sciences Program under contract number DE-SC0012704 (KC0401040).

\section{References}

Afonine, P. V., Grosse-Kunstleve, R. W., Echols, N., Headd, J. J., Moriarty, N. W., Mustyakimov, M., Terwilliger, T. C., Urzhumtsev, A., Zwart, P. H. \& Adams, P. D. (2012). Acta Cryst. D68, 352-367. Akey, D. L., Brown, W. C., Dutta, S., Konwerski, J., Jose, J., Jurkiw, T. J., DelProposto, J., Ogata, C. M., Skiniotis, G., Kuhn, R. J. \& Smith, J. L. (2014). Science, 343, 881-885.

Axford, D., Foadi, J., Hu, N.-J., Choudhury, H. G., Iwata, S., Beis, K., Evans, G. \& Alguel, Y. (2015). Acta Cryst. D71, 1228-1237.

Barends, T. R., Foucar, L., Botha, S., Doak, R. B., Shoeman, R. L., Nass, K., Koglin, J. E., Williams, G. J., Boutet, S., Messerschmidt, M. \& Schlichting, I. (2014). Nature, 505, 244-247.

Basu, S., Kaminski, J. W., Panepucci, E., Huang, C.-Y., Warshamanage, R., Wang, M. \& Wojdyla, J. A. (2019). J. Synchrotron Rad. 26, 244-252.

Batyuk, A., Galli, L., Ishchenko, A., Han, G. W., Gati, C., Popov, P. A., Lee, M. Y., Stauch, B., White, T. A., Barty, A., Aquila, A., Hunter, M. S., Liang, M. N., Boutet, S., Pu, M. C., Liu, Z. J., Nelson, G., James, D., Li, C. F., Zhao, Y., Spence, J. C. H., Liu, W., Fromme, P., Katritch, V., Weierstall, U., Stevens, R. C. \& Cherezov, V. (2016). Sci. Adv. 2, e1600292.

Beyerlein, K. R., Dierksmeyer, D., Mariani, V., Kuhn, M., Sarrou, I., Ottaviano, A., Awel, S., Knoska, J., Fuglerud, S., Jönsson, O., Stern, S., Wiedorn, M. O., Yefanov, O., Adriano, L., Bean, R., Burkhardt, A., Fischer, P., Heymann, M., Horke, D. A., Jungnickel, K. E. J., Kovaleva, E., Lorbeer, O., Metz, M., Meyer, J., Morgan, A., Pande, K., Panneerselvam, S., Seuring, C., Tolstikova, A., Lieske, J., Aplin, S., Roessle, M., White, T. A., Chapman, H. N., Meents, A. \& Oberthuer, D. (2017). IUCrJ, 4, 769-777.

Botha, S., Nass, K., Barends, T. R. M., Kabsch, W., Latz, B., Dworkowski, F., Foucar, L., Panepucci, E., Wang, M., Shoeman, R. L., Schlichting, I. \& Doak, R. B. (2015). Acta Cryst. D71, 387-397. Boutet, S., Lomb, L., Williams, G. J., Barends, T. R., Aquila, A., Doak, R. B., Weierstall, U., DePonte, D. P., Steinbrener, J., Shoeman, R. L., Messerschmidt, M., Barty, A., White, T. A., Kassemeyer, S., Kirian, R. A., Seibert, M. M., Montanez, P. A., Kenney, C., Herbst, R., Hart, P., Pines, J., Haller, G., Gruner, S. M., Philipp, H. T., Tate, M. W., Hromalik, M., Koerner, L. J., van Bakel, N., Morse, J., Ghonsalves, W., Arnlund, D., Bogan, M. J., Caleman, C., Fromme, R., Hampton, C. Y., Hunter, M. S., Johansson, L. C., Katona, G., Kupitz, C., Liang, M., Martin, A. V., Nass, K., Redecke, L., Stellato, F., Timneanu, N., Wang, D., Zatsepin, N. A., Schafer, D., Defever, J., Neutze, R., Fromme, P., Spence, J. C., Chapman, H. N. \& Schlichting, I. (2012). Science, 337, 362-364.

Broennimann, Ch., Eikenberry, E. F., Henrich, B., Horisberger, R., Huelsen, G., Pohl, E., Schmitt, B., Schulze-Briese, C., Suzuki, M., Tomizaki, T., Toyokawa, H. \& Wagner, A. (2006). J. Synchrotron Rad. 13, 120-130.

Bunkóczi, G., McCoy, A. J., Echols, N., Grosse-Kunstleve, R. W., Adams, P. D., Holton, J. M., Read, R. J. \& Terwilliger, T. C. (2015). Nat. Methods, 12, 127-130.

Chapman, H. N., Fromme, P., Barty, A., White, T. A., Kirian, R. A., Aquila, A., Hunter, M. S., Schulz, J., DePonte, D. P., Weierstall, U., Doak, R. B., Maia, F. R., Martin, A. V., Schlichting, I., Lomb, L., Coppola, N., Shoeman, R. L., Epp, S. W., Hartmann, R., Rolles, D., Rudenko, A., Foucar, L., Kimmel, N., Weidenspointner, G., Holl, P., Liang, M., Barthelmess, M., Caleman, C., Boutet, S., Bogan, M. J., Krzywinski, J., Bostedt, C., Bajt, S., Gumprecht, L., Rudek, B., Erk, B., Schmidt, C., Hömke, A., Reich, C., Pietschner, D., Strüder, L., Hauser, G., Gorke, H., Ullrich, J., Herrmann, S., Schaller, G., Schopper, F., Soltau, H., Kühnel, K. U., Messerschmidt, M., Bozek,
J. D., Hau-Riege, S. P., Frank, M., Hampton, C. Y., Sierra, R. G., Starodub, D., Williams, G. J., Hajdu, J., Timneanu, N., Seibert, M. M., Andreasson, J., Rocker, A., Jönsson, O., Svenda, M., Stern, S., Nass, K., Andritschke, R., Schröter, C. D., Krasniqi, F., Bott, M., Schmidt, K. E., Wang, X., Grotjohann, I., Holton, J. M., Barends, T. R., Neutze, R., Marchesini, S., Fromme, R., Schorb, S., Rupp, D., Adolph, M., Gorkhover, T., Andersson, I., Hirsemann, H., Potdevin, G., Graafsma, H., Nilsson, B. \& Spence, J. C. (2011). Nature, 470, 73-77.

Chen, V. B., Arendall, W. B., Headd, J. J., Keedy, D. A., Immormino, R. M., Kapral, G. J., Murray, L. W., Richardson, J. S. \& Richardson, D. C. (2010). Acta Cryst. D66, 12-21.

Coquelle, N., Brewster, A. S., Kapp, U., Shilova, A., Weinhausen, B., Burghammer, M. \& Colletier, J.-P. (2015). Acta Cryst. D71, 11841196.

Cowtan, K. (2006). Acta Cryst. D62, 1002-1011.

Cowtan, K. D. \& Zhang, K. Y. J. (1999). Prog. Biophys. Mol. Biol. 72, 245-270.

Dauter, Z. (2006). Acta Cryst. D62, 867-876.

Dauter, Z., Dauter, M., de La Fortelle, E., Bricogne, G. \& Sheldrick, G. M. (1999). J. Mol. Biol. 289, 83-92.

Diederichs, K. \& Wang, M. (2017). Methods Mol. Biol. 1607, 239-272.

Doutch, J., Hough, M. A., Hasnain, S. S. \& Strange, R. W. (2012). J. Synchrotron Rad. 19, 19-29.

Echols, N., Morshed, N., Afonine, P. V., McCoy, A. J., Miller, M. D., Read, R. J., Richardson, J. S., Terwilliger, T. C. \& Adams, P. D. (2014). Acta Cryst. D70, 1104-1114.

Evans, G., Axford, D., Waterman, D. \& Owen, R. L. (2011). Crystallogr. Rev. 17, 105-142.

Evans, P. R. \& Murshudov, G. N. (2013). Acta Cryst. D69, 1204-1214.

Flot, D., Mairs, T., Giraud, T., Guijarro, M., Lesourd, M., Rey, V., van Brussel, D., Morawe, C., Borel, C., Hignette, O., Chavanne, J., Nurizzo, D., McSweeney, S. \& Mitchell, E. (2010). J. Synchrotron Rad. 17, 107-118.

Foadi, J., Aller, P., Alguel, Y., Cameron, A., Axford, D., Owen, R. L., Armour, W., Waterman, D. G., Iwata, S. \& Evans, G. (2013). Acta Cryst. D69, 1617-1632.

Fuchs, M. R., Bhogadi, D. K., Jakoncic, J., Myers, S., Sweet, R. M., Berman, L. E., Skinner, J., Idir, M., Chubar, O., McSweeney, S. \& Schneider, D. K. (2016). AIP Conf. Proc. 1741, 0300026.

Gao, Y., Xu, W., Shi, W., Soares, A., Jakoncic, J., Myers, S., Martins, B., Skinner, J., Liu, Q., Bernstein, H., McSweeney, S., Nazaretski, E. \& Fuchs, M. R. (2018). J. Synchrotron Rad. 25, 1362-1370.

Garman, E. F. (2014). Science, 343, 1102-1108.

Garman, E. F. \& Weik, M. (2017). J. Synchrotron Rad. 24, 1-6.

Gati, C., Bourenkov, G., Klinge, M., Rehders, D., Stellato, F., Oberthür, D., Yefanov, O., Sommer, B. P., Mogk, S., Duszenko, M., Betzel, C., Schneider, T. R., Chapman, H. N. \& Redecke, L. (2014). IUCrJ, 1, 87-94.

Giordano, R., Leal, R. M. F., Bourenkov, G. P., McSweeney, S. \& Popov, A. N. (2012). Acta Cryst. D68, 649-658.

Guo, G., Fuchs, M. R., Shi, W., Skinner, J., Berman, E., Ogata, C. M., Hendrickson, W. A., McSweeney, S. \& Liu, Q. (2018). IUCrJ, 5, 238-246.

Henderson, R. (1990). Proc. R. Soc. London Ser. B, 241, 6-8.

Hendrickson, W. A. (2014). Q. Rev. Biophys. 47, 49-93.

Hendrickson, W. A. \& Teeter, M. M. (1981). Nature, 290, 107-113.

Holton, J. M. \& Frankel, K. A. (2010). Acta Cryst. D66, 393-408.

Huang, C.-Y., Olieric, V., Howe, N., Warshamanage, R., Weinert, T., Panepucci, E., Vogeley, L., Basu, S., Diederichs, K., Caffrey, M. \& Wang, M. (2018). Commun. Biol. 1, 124.

Hunter, M. S., Yoon, C. H., DeMirci, H., Sierra, R. G., Dao, E. H., Ahmadi, R., Aksit, F., Aquila, A. L., Ciftci, H., Guillet, S., Hayes, M. J., Lane, T. J., Liang, M., Lundström, U., Koglin, J. E., Mgbam, P., Rao, Y., Zhang, L., Wakatsuki, S., Holton, J. M. \& Boutet, S. (2016). Nat. Commun. 7.

Ji, X. Y., Sutton, G., Evans, G., Axford, D., Owen, R. \& Stuart, D. I. (2010). EMBO J. 29, 505-514. 
La Fortelle, E. de \& Bricogne, G. (1997). Methods Enzymol. 276, 472494.

Laskowski, R. A., MacArthur, M. W., Moss, D. S. \& Thornton, J. M. (1993). J. Appl. Cryst. 26, 283-291.

Liebschner, D., Yamada, Y., Matsugaki, N., Senda, M. \& Senda, T. (2016). Acta Cryst. D72, 728-741.

Liu, Q., Dahmane, T., Zhang, Z., Assur, Z., Brasch, J., Shapiro, L., Mancia, F. \& Hendrickson, W. A. (2012). Science, 336, 1033-1037.

Liu, Q., Guo, Y., Chang, Y., Cai, Z., Assur, Z., Mancia, F., Greene, M. I. \& Hendrickson, W. A. (2014). Acta Cryst. D70, 2544-2557.

Liu, Q. \& Hendrickson, W. A. (2015). Curr. Opin. Struct. Biol. 34, 99107.

Liu, Q. \& Hendrickson, W. A. (2017). In Protein Crystallography, Methods in Molecular Biology, Vol. 1607, 377-399.

Liu, Q., Liu, Q. \& Hendrickson, W. A. (2013). Acta Cryst. D69, 13141332.

Liu, Q., Zhang, Z. \& Hendrickson, W. A. (2011). Acta Cryst. D67, 4559.

Liu, Z. J., Vysotski, E. S., Vysotski, E. S., Chen, C., Rose, J. P., Lee, J. \& Wang, B. (2000). Protein Sci. 9, 2085-2093.

Martin-Garcia, J. M., Conrad, C. E., Nelson, G., Stander, N., Zatsepin, N. A., Zook, J., Zhu, L., Geiger, J., Chun, E., Kissick, D., Hilgart, M. C., Ogata, C., Ishchenko, A., Nagaratnam, N., Roy-Chowdhury, S., Coe, J., Subramanian, G., Schaffer, A., James, D., Ketwala, G., Venugopalan, N., Xu, S., Corcoran, S., Ferguson, D., Weierstall, U., Spence, J. C. H., Cherezov, V., Fromme, P., Fischetti, R. F. \& Liu, W. (2017). IUCrJ, 4, 439-454.

Meents, A., Wiedorn, M. O., Srajer, V., Henning, R., Sarrou, I., Bergtholdt, J., Barthelmess, M., Reinke, P. Y. A., Dierksmeyer, D., Tolstikova, A., Schaible, S., Messerschmidt, M., Ogata, C. M., Kissick, D. J., Taft, M. H., Manstein, D. J., Lieske, J., Oberthuer, D., Fischetti, R. F. \& Chapman, H. N. (2017). Nat. Commun. 8, 1281.

Melnikov, I., Polovinkin, V., Kovalev, K., Gushchin, I., Shevtsov, M., Shevchenko, V., Mishin, A., Alekseev, A., Rodriguez-Valera, F., Borshchevskiy, V., Cherezov, V., Leonard, G. A., Gordeliy, V. \& Popov, A. (2017). Sci. Adv. 3, e1602952.

Melnikov, I., Svensson, O., Bourenkov, G., Leonard, G. \& Popov, A. (2018). Acta Cryst. D74, 355-365.

Mueller-Dieckmann, C., Panjikar, S., Tucker, P. A. \& Weiss, M. S. (2005). Acta Cryst. D61, 1263-1272.

Nakane, T., Song, C., Suzuki, M., Nango, E., Kobayashi, J., Masuda, T., Inoue, S., Mizohata, E., Nakatsu, T., Tanaka, T., Tanaka, R., Shimamura, T., Tono, K., Joti, Y., Kameshima, T., Hatsui, T., Yabashi, M., Nureki, O., Iwata, S. \& Sugahara, M. (2015). Acta Cryst. D71, 2519-2525.

Nass, K., Meinhart, A., Barends, T. R. M., Foucar, L., Gorel, A., Aquila, A., Botha, S., Doak, R. B., Koglin, J., Liang, M., Shoeman, R. L., Williams, G., Boutet, S. \& Schlichting, I. (2016). IUCrJ, 3, 180-191.

Nogly, P., James, D., Wang, D., White, T. A., Zatsepin, N., Shilova, A., Nelson, G., Liu, H., Johansson, L., Heymann, M., Jaeger, K., Metz, M., Wickstrand, C., Wu, W., Båth, P., Berntsen, P., Oberthuer, D.,
Panneels, V., Cherezov, V., Chapman, H., Schertler, G., Neutze, R., Spence, J., Moraes, I., Burghammer, M., Standfuss, J. \& Weierstall, U. (2015). IUCrJ, 2, 168-176.

Perrakis, A., Cipriani, F., Castagna, J.-C., Claustre, L., Burghammer, M., Riekel, C. \& Cusack, S. (1999). Acta Cryst. D55, 1765-1770.

Read, R. J. \& McCoy, A. J. (2011). Acta Cryst. D67, 338-344.

Rose, J. P., Wang, B.-C. \& Weiss, M. S. (2015). IUCrJ, 2, 431-440.

Rose, J. P. \& Wang, B. C. (2016). Arch. Biochem. Biophys. 602, 80-94. Sheldrick, G. M. (2010). Acta Cryst. D66, 479-485.

Smith, J. L., Fischetti, R. F. \& Yamamoto, M. (2012). Curr. Opin. Struct. Biol. 22, 602-612.

Stellato, F., Oberthür, D., Liang, M., Bean, R., Gati, C., Yefanov, O., Barty, A., Burkhardt, A., Fischer, P., Galli, L., Kirian, R. A., Meyer, J., Panneerselvam, S., Yoon, C. H., Chervinskii, F., Speller, E., White, T. A., Betzel, C., Meents, A. \& Chapman, H. N. (2014). IUCrJ, 1, 204-212.

Terwilliger, T. C., Bunkóczi, G., Hung, L.-W., Zwart, P. H., Smith, J. L., Akey, D. L. \& Adams, P. D. (2016). Acta Cryst. D72, 359-374.

Vonrhein, C., Blanc, E., Roversi, P. \& Bricogne, G. (2007). Methods Mol. Biol. 364, 215-230.

Wagner, A., Duman, R., Henderson, K. \& Mykhaylyk, V. (2016). Acta Cryst. D72, 430-439.

Waterman, D. G., Winter, G., Gildea, R. J., Parkhurst, J. M., Brewster, A. S., Sauter, N. K. \& Evans, G. (2016). Acta Cryst. D72, 558-575.

Weinert, T., Olieric, V., Waltersperger, S., Panepucci, E., Chen, L., Zhang, H., Zhou, D., Rose, J., Ebihara, A., Kuramitsu, S., Li, D., Howe, N., Schnapp, G., Pautsch, A., Bargsten, K., Prota, A. E., Surana, P., Kottur, J., Nair, D. T., Basilico, F., Cecatiello, V., Pasqualato, S., Boland, A., Weichenrieder, O., Wang, B. C., Steinmetz, M. O., Caffrey, M. \& Wang, M. (2015). Nat. Methods, 12, 131-133.

Yamamoto, M., Hirata, K., Yamashita, K., Hasegawa, K., Ueno, G., Ago, H. \& Kumasaka, T. (2017). IUCrJ, 4, 529-539.

Yamashita, K., Hirata, K. \& Yamamoto, M. (2018). Acta Cryst. D74, 441-449.

Yamashita, K., Kuwabara, N., Nakane, T., Murai, T., Mizohata, E., Sugahara, M., Pan, D., Masuda, T., Suzuki, M., Sato, T., Kodan, A., Yamaguchi, T., Nango, E., Tanaka, T., Tono, K., Joti, Y., Kameshima, T., Hatsui, T., Yabashi, M., Manya, H., Endo, T., Kato, R., Senda, T., Kato, H., Iwata, S., Ago, H., Yamamoto, M., Yumoto, F. \& Nakatsu, T. (2017). IUCrJ, 4, 639-647.

Yang, C., Pflugrath, J. W., Courville, D. A., Stence, C. N. \& Ferrara, J. D. (2003). Acta Cryst. D59, 1943-1957.

Zander, U., Bourenkov, G., Popov, A. N., de Sanctis, D., Svensson, O., McCarthy, A. A., Round, E., Gordeliy, V., Mueller-Dieckmann, C. \& Leonard, G. A. (2015). Acta Cryst. D71, 2328-2343.

Zander, U., Cianci, M., Foos, N., Silva, C. S., Mazzei, L., Zubieta, C., de Maria, A. \& Nanao, M. H. (2016). Acta Cryst. D72, 1026-1035.

Zeldin, O. B., Brockhauser, S., Bremridge, J., Holton, J. M. \& Garman, E. F. (2013a). Proc. Natl Acad. Sci. USA, 110, 20551-20556.

Zeldin, O. B., Gerstel, M. \& Garman, E. F. (2013b). J. Appl. Cryst. 46, 1225-1230. 